\title{
An observational study of GMCs in the Magellanic Clouds with the $A S T E$ telescope
}

\author{
Tetsuhiro Minamidani ${ }^{1}$, Norikazu Mizuno ${ }^{2}$, Yoji Mizuno ${ }^{2}$, \\ Akiko Kawamura $^{2}$, Toshikazu Onishi ${ }^{2}$, Ken'ichi Tatematsu ${ }^{3}$, \\ Tetsuo Hasegawa ${ }^{3}$, Masafumi Ikeda ${ }^{4}$ and Yasuo Fukui ${ }^{2}$ \\ ${ }^{1}$ Department of Physics, Faculty of Science, Hokkaido University N10W8, Kita-ku, \\ Sapporo, 060-0810, Japan \\ email: tetsu@astro1.sci.hokudai.ac.jp \\ ${ }^{2}$ Department of Astrophysics, Nagoya University Furo-cho, Chikusa-ku, \\ Nagoya 464-8602, Japan \\ ${ }^{3}$ National Astronomical Observatory of Japan Mitaka, Tokyo 181-8588, Japan \\ ${ }^{4}$ Research Center for the Early Universe and Department of Physics, University of Tokyo \\ Tokyo 113-0033, Japan
}

\begin{abstract}
We report the results of the submillimeter observations with the ASTE $10 \mathrm{~m}$ telescope toward the giant molecular clouds (GMCs) in the Magellanic Clouds to reveal the physical properties of dense molecular gas, the principle sites of star and cluster formation. Six GMCs in the Large Magellanic Cloud have been mapped in the ${ }^{12} \mathrm{CO}(J=3-2)$ transition and 32 clumps are identified in these GMCs at a resolution of 5 pc. These data are combined with ${ }^{12} \mathrm{CO}(J=1-0)$ and ${ }^{13} \mathrm{CO}(J=1-0)$ results and compared with $\mathrm{LVG}$ calculations to derive the density and temperature of clumps. The derived density and temperature are distributed in wide ranges. We have made small mapping observations in the ${ }^{13} \mathrm{CO}(J=3-2)$ transition toward 9 representative peak positions of clumps to determine the density and temperature of clumps. These physical properties are constrained well and there are differences in density and temperature among clumps. We suggest that these differences of clump properties represent an evolutionary sequence of GMCs in terms of density increase leading to star formation.
\end{abstract}

Keywords. ISM: clouds, ISM: molecules, galaxies: individual (LMC), Magellanic Clouds, radio lines: ISM, submillimeter

\section{Introduction}

Giant molecular clouds (GMCs) are important as sites of star and cluster formation. GMCs consist mainly of molecular hydrogen $\left(\mathrm{H}_{2}\right)$, which does not have a permanent electric dipole moment. This means a lack of appropriate emission lines excited under the typical condition of GMCs. Carbon monoxide, CO, is the second most abundant molecule and has rotational transitions excited under the typical condition in GMCs. These CO transitions are mainly used to trace molecular components in the interstellar medium.

The observations of NANTEN 4-m telescope in $2.6 \mathrm{~mm}{ }^{12} \mathrm{CO}(J=1-0)$ emission reveal the distribution of GMCs in the whole Large Magellanic Cloud (LMC), at 40 pc resolution (e.g., Fukui et al. 2008). Fukui et al. (2008) identified 272 GMCs. These GMCs are classified into 3 types in terms of massive star formation activity; Type I shows no signs of massive star formation, Type II is associated with H II regions, and Type III is associated with both HII regions and young clusters (e.g., Kawamura et al. 2008). 
Kawamura et al. (2008) suggest that these GMC types correspond to the evolutionary stages of GMCs.

Observations of higher transitions $(J=2-1, J=3-2, J=4-3, J=7-6)$ of CO spectra of GMCs suggested that there is either warm gas or dense gas, or both in GMCs (e.g., Sorai et al. 2001; Johansson et al. 1998; Heikkilä et al. 1999; Bolatto et al. 2005; Israel et al. 2003; Kim et al. 2004; Kim 2006; Pineda et al. 2008; Minamidani et al. 2008). We aim to obtain submillimeter molecular data at better signal-to-noise ratios than in previous studies to estimate temperatures and densities of molecular gas in the GMCs. We combine the ${ }^{12} \mathrm{CO}(J=3-2)$ and ${ }^{13} \mathrm{CO}(J=3-2)$ data observed with the ASTE telescope, and the ${ }^{12} \mathrm{CO}(J=1-0)$ and ${ }^{13} \mathrm{CO}(J=1-0)$ data obtained with $S E S T$ and MOPRA telescopes.

\section{Observations and results}

Observations of ${ }^{12} \mathrm{CO}(J=3-2)$ and ${ }^{13} \mathrm{CO}(J=3-2)$ transitions at $345 \mathrm{GHz}$ and 330 $\mathrm{GHz}$, respectively, were made with the ASTE 10-m telescope at Pampa la Bola in Chile. The details of ${ }^{12} \mathrm{CO}(J=3-2)$ observations and results are described by Minamidani et al. (2008). ${ }^{13} \mathrm{CO}(J=3-2)$ observations were made using the single "cartridge type" SIS receiver (SC345), and the spectrometer was XF-type digital autocorrelator. The halfpower beam width was $23^{\prime \prime}$ at $330 \mathrm{GHz}$, and this corresponds to $5.6 \mathrm{pc}$ at the distance of the LMC, $50 \mathrm{kpc}$. We have made $3 \times 3$ points mapping observations with $20^{\prime \prime}$ grid toward 9 representative peaks of ${ }^{12} \mathrm{CO}(J=3-2)$ clumps observed by Minamidani et al. (2008). They were 30 Dor No.1, No.2, No.3, No.4, N 159 No.1, No.2, No.4, N 206D No.1, and GMC 225 No.1. The clumps in the 30 Dor and the N 159 regions are in Type III GMCs, the clump in the N 206D region is in Type II GMC, and the clump in the GMC 225 region is in Type I GMC. Figure 1 shows the results of the N 159 region.

\section{LVG analysis}

To estimate the physical properties of the molecular gas, we have performed the LVG (Large Velocity Gradient) analysis (Goldreich \& Kwan 1974) of the CO rotational transitions. The LVG radiative transfer code simulates a spherically symmetric cloud of constant density, temperature, and velocity gradient. It solves the equations of statistical equilibrium for the fractional population of $\mathrm{CO}$ rotational levels at given density and temperature. It includes the lowest 40 rotational levels and uses the Einstein's A and collisional coefficients of Schöier et al. (2005). Calculated ranges are 5-200 K in kinetic temperature and $10-10^{6} \mathrm{~cm}^{-3}$ in density of molecular hydrogen.

Figure 2 shows the results of LVG analysis for reference. This indicates that 2 intensity ratios, ${ }^{13} \mathrm{CO}(J=3-2)$ to ${ }^{13} \mathrm{CO}(J=1-0)$ and ${ }^{12} \mathrm{CO}(J=3-2)$ to ${ }^{13} \mathrm{CO}(J=3-2)$, are orthogonal in wide ranges of density and temperature as compared with the ratios of ${ }^{12} \mathrm{CO}(J=3-2)$ to ${ }^{12} \mathrm{CO}(J=1-0)$ and ${ }^{12} \mathrm{CO}(J=1-0)$ to ${ }^{13} \mathrm{CO}(J=1-0)$ (See Figure 19 of Minamidani et al. 2008). Figure 2 also shows the results of LVG analysis of representative 3 clumps, 30 Dor No.1, N 206D No.1, and GMC 225 No.1.

The results of LVG analysis for studied clumps are presented in Figure 3. The physical properties of these clumps are well constrained as compared with previous analysis using the ratios of ${ }^{12} \mathrm{CO}(J=3-2)$ to ${ }^{12} \mathrm{CO}(J=1-0)$ and ${ }^{12} \mathrm{CO}(J=1-0)$ to ${ }^{13} \mathrm{CO}(J=1-0)$ (See Figure 21 of Minamidani et al. 2008). 


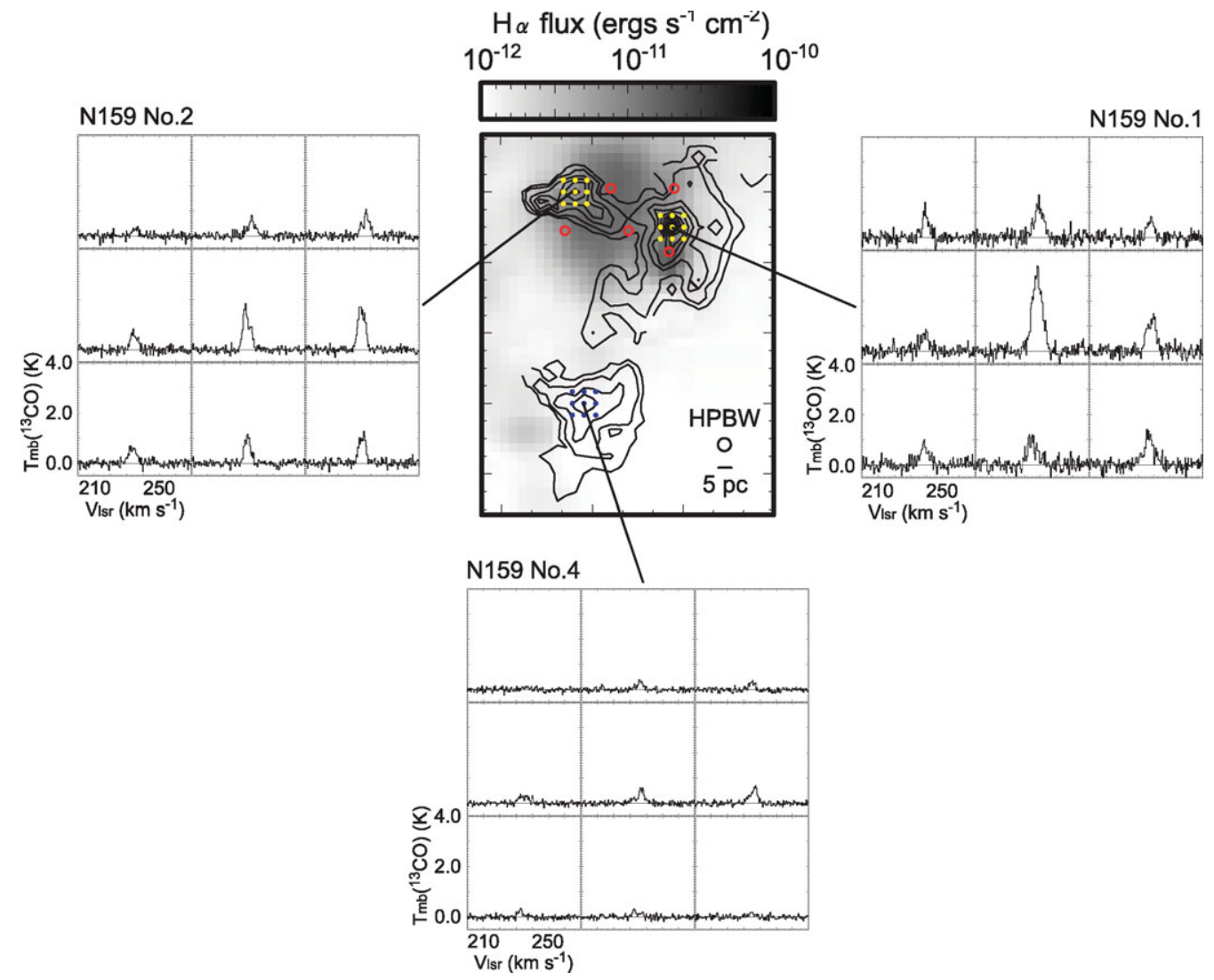

Figure 1. ${ }^{13} \mathrm{CO}(J=3-2)$ spectra in the $\mathrm{N} 159$ region. Black and white image is $\mathrm{H} \alpha$, contours are ${ }^{12} \mathrm{CO}(J=3-2)$ integrated intensity, and red circles indicate positions of young clusters $\left(<10 \mathrm{Myr}\right.$, SWB0, Minamidani et al. 2008). Positions observed in ${ }^{13} \mathrm{CO}(J=3-2)$ are indicated by yellow and blue dots.

\section{Evolution of GMCs}

The results of the LVG analysis indicate that clumps are distributed $\sim 7 \times 10^{2}-$ $1.5 \times 10^{5} \mathrm{~cm}^{-3}$ in density and $15-200 \mathrm{~K}$ in temperature. These differences of clump density and temperature generally correspond to the types of GMCs; a clump in the Type I GMC is cool and less dense, and clumps in the Type III GMCs are warm and dense. We should note that the properties of clumps are different even in the same GMC. It seems that the properties of clumps depend on the stage of evolution and are affected by the local environments.

\section{Acknowledgements}

A part of this study was financially supported by MEXT Grant-in-Aid for Scientific Research on Priority Area (No. 15071202 and No. 15071203) and by JSPS (No. 14102003, core-to-core program 17004, and No. 18684003). The ASTE project is driven by Nobeyama Radio Observatory (NRO), a branch of National Astronomical Observatory of Japan (NAOJ), in collaboration with University of Chile, and Japanese institutes including University of Tokyo, Nagoya University, Osaka Prefecture University, Ibaraki University, Kobe University, and Hokkaido University. We are grateful to all the members of $A S T E$ team. 


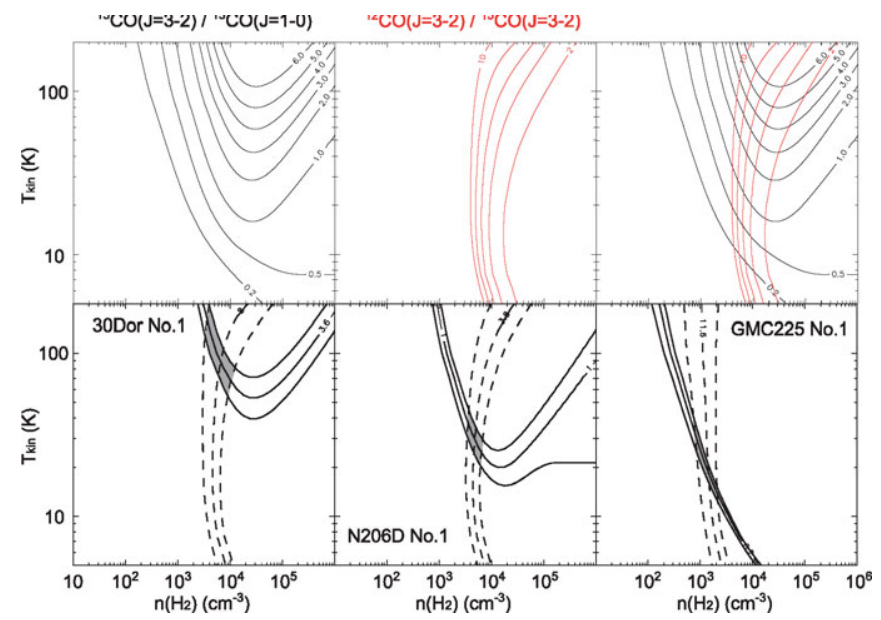

Figure 2. Contour plots of LVG analysis for (upper panels) reference and (lower panels) representative 3 clumps. The vertical axis is kinetic temperature, $T_{\text {kin }}$, and the horizontal axis is molecular hydrogen density, $n\left(\mathrm{H}_{2}\right)$. Solid lines are the ratios of ${ }^{13} \mathrm{CO}(J=3-2)$ to ${ }^{13} \mathrm{CO}(J=3-2)$ and dashed lines are the ratios of ${ }^{12} \mathrm{CO}(J=3-2)$ to ${ }^{13} \mathrm{CO}(J=3-2)$. Hatched areas are the regions in which these two ratios overlap within errors.

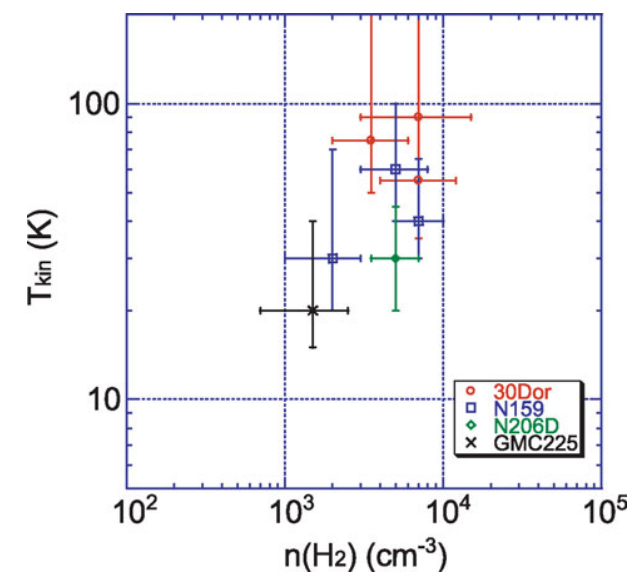

Figure 3. Plot of LVG results. The vertical axis is kinetic temperature, $T_{\text {kin }}$, and the horizontal axis is molecular hydrogen density, $n\left(\mathrm{H}_{2}\right)$.

\section{References}

Bolatto, A. D., Israel, F. P., \& Martin, C. L. 2005, ApJ, 633, 210

Fukui, Y., Kawamura, A., Minamidani, T., et al. 2008, ApJS, 178, 56

Goldreich, P. \& Kwan, J. 1974, ApJ, 189, 441

Heikkilä, A., Johansson, L. E. B., \& Olofsson, H. 1999, A\&A, 344, 817

Israel, F. P., Johansson, L. E. B., Rubio, M., et al. 2003, A\&BA, 406, 817

Johansson, L. E. B., Greve, A., Booth, R. S., et al. 1998, A\& A, 331, 857

Kawamura, A., et al. 2008, ApJS, submitted

Kim, S., Walsh, W., \& Xiao, K. 2004, ApJ, 616, 865

Kim, S. 2006, PASP, 118, 94

Minamidani, T., Mizuno, N., Mizuno, Y., et al. 2008, ApJS, 175, 485

Pineda, J. L., Mizuno, N., Stutzki, J., et al. 2008, A\&BA, 482, 197

Schöier, F. L., van der Tak, F. F. S., van Dichoeck, E. F., \& Black, J. H. 2005, AछAA, 432, 369

Sorai, K., Hasegawa, T., Booth, R. S., et al. 2001, ApJ, 551, 794 


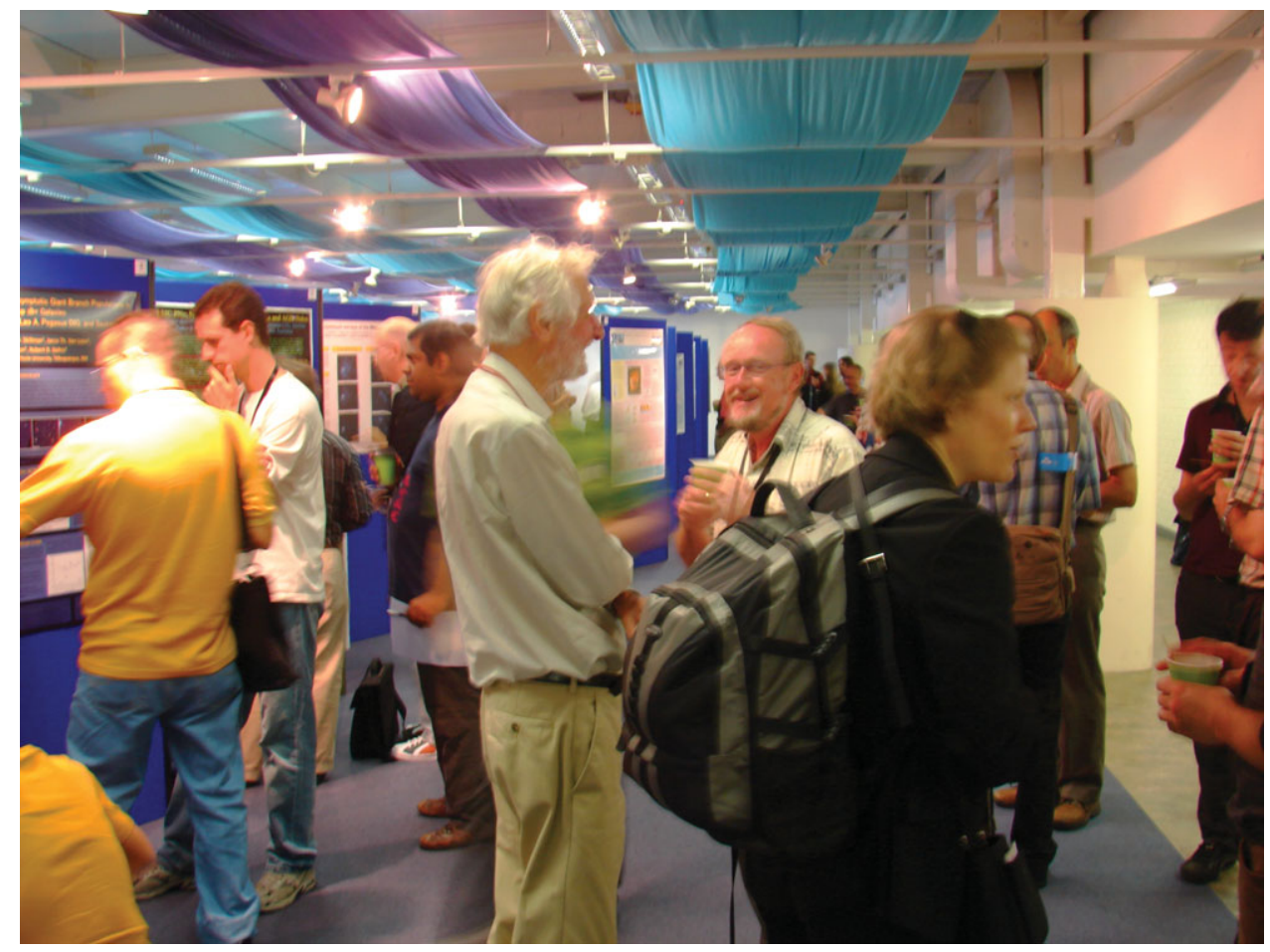

Poster area, frequented by the curious and those enjoying a conversation — and coffee. 